\title{
Comportamento da artéria ovárica em éguas sem raça definida (Equuscaballus, Linnaeus, 1758)
}

\author{
Behavior of the ovaric artery in crossbred mares (Equus caballus, Linnaeus, 1758)
}

\author{
Lindolfo Gonçalves Cabral, Patricia Reginato Facciotti, Dulcinéa Gonçalves Teixeira, \\ Karla Patrícia Cardoso Araújo, Daniele dos Santos Martins, Rose Eli Grassi Rici, \\ Carlos Eduardo Ambrósio, Maria Angelica Miglino \& Arani Nanci Bomfim Mariana
}

\begin{abstract}
RESUMO
Atualmente, na medicina veterinária, os estudos envolvendo reprodução de eqüinos e principalmente a qualidade das fêmeas, que na maioria das vezes são consideradas como receptoras, são de extrema valia, devido a ganho de produção e qualidade de plantel dos criadores, sendo assim, teve-se por objetivo estudar o comportamento da artéria ovárica em éguas, ou seja, a sua ramificação e distribuição no parênquima ovariano, para dar subsídios a um melhor entendimento das manobras de melhoramento genético e produção, focando aspectos morfológicos e fisiológicos da vascularização e viabilidade deste órgão na reprodução animal. Foram utilizados 68 ovários (34 pares) de éguas adultas sem raça definida, com diferentes idades, obtidas no Frigorífico Pomar, município de Araguari, MG. Verificou-se que a artéria ovárica apresenta um trajeto longo, flexuoso e espiralado, alcançando a glândula por meio da margem mesovárica, próximo à extremidade uterina. Esta artéria mantém o sentido ora dorsal ora ventral, segue em direção à extremidade tubárica, contorna-a até atingir a fossa ovárica, e penetra na glândula somente após ter percorrido toda a sua superfície. A artéria ovárica apresentou dois arranjos vasculares: um com emissão de 2 a 62 ramos dorsais e 4 a 46 ramos ventrais, durante seu percurso na margem mesovárica em 51 preparações (75\%) e no outro arranjo a artéria ovárica, na extremidade uterina, dividiu-se em um ramo dorsal e outro ventral em 17 preparações (25\%). Em relação às faces dos ovários, a face lateral apresentou um número maior de ramos que a face medial. Nos ovários direitos, os quadrantes mais irrigados foram, por ordem: o dorsocranial, o ventrocranial, o dorsocaudal e o ventrocaudal. Nos ovários esquerdos, foram: o ventrocranial, o dorsocranial, o ventrocaudal e o dorsocaudal.
\end{abstract}

Descritores: anatomia microscópica, éguas, Equus caballus, ovário, artérias.

\section{ABSTRACT}

Actually, on veterinary medicine, studies related to equine reproduction and mainly female quality, that most of the time, are considerate carrier production females and have an extremely value due to production increase and breed quality came from the farmers, then the aiming of the research was to study the ovaric artery behavior in mares, related with a fine description of ramification and distribution at ovarian parenchyma for further basal studies on reproduction field and productions rates correlates to reproductive performance, focusing morphological and physiological features from this organ vascularization and viability on animal reproduction. Sixty eight ovaries from adult crossbred mares of different ages obtained at the Slaughterhouse Pomar, Araguari - MG, Brazil. It was observed that the ovaric artery presents a long, sinuous and spiral course, and reaches the ovarian gland though the mesovaric margin near the extremity of the uterus, running either in a dorsal or ventral position. Then it runs towards the end of the uterine tube, circling it until it reach the ovarian fossae, and penetrate the ovarian gland only after passing along its entire surface. The ovaric artery presented two vascular arrangements: (1) one emits 2 to 62 dorsal branches and 4 to 46 ventral branches during its course along the mesovaric margin in 51 specimens (75\%); (2) the other at the extremity of the uterus, is divided into a dorsal and ventral branches as was observed in 17 specimens $(25 \%)$. Regarding the ovaric surfaces, the lateral surface presents a greater number of branches than the medial surface. In the right ovaries, the most highly irrigated quadrant was, in decreasing order, the dorsocranial, the ventrocranial, the dorsocaudal, and the ventrocaudal. In the left ovaries, the most irrigated was, in decreasing order, the ventrocranial, the dorsocranial, the ventrocaudal, and the dorsocaudal quadrants.

Key words: macroscopic anatomy, mares, Equus caballus, ovaries, arteries. 


\section{INTRODUÇÃO}

A área da reprodução animal apresenta crescentes avanços, porem há escassez de literatura específica referente à vascularização do ovário, dessa forma é imprescindível fornecer subsídios para interpretações morfofuncionais deste órgão e, portanto um entendimento mais preciso das manobras de melhoramento genético.

A artéria ovárica emerge diretamente da aorta abdominal, desce em direção ventro-caudal e se divide em ramos ovárico e uterino, sendo o último o principal suprimento arterial do útero [8,13,17,34,35,37]. $\mathrm{O}$ ramo uterino ramifica-se nos cornos do útero e o ramo ovárico se distribui no parênquima do ovário [25].

As artérias do ramo uterino são tortuosas e de parede espessa, penetrando pela porção cranial do ligamento largo formando uma camada vascular de grandes vasos, arranjados em forma de círculo entre as camadas musculares. As veias acompanham as artérias e formam plexos pampiniformes [3,13].

A artéria ovariana divide-se em uma série de ramos que penetram no ovário pelo hilo, ao longo da margem dorsal. A égua apresenta uma exceção notável: a organogênese da glândula caracteriza-se pela extensão da invasão peritoneal, que recebe uma pseudoalbugínea espessa. Não existe hilo e a artéria ovariana penetra no órgão após ter percorrido a superfície [4].

Os dois ramos principais da artéria ovárica se ramificam em ramos de primeira ordem em direção ao fundo, que por sua vez também se dividem em ramos fortemente torcidos de segunda ordem [31].

Teve-se por objetivo estudar o comportamento da artéria ovárica na égua, ou seja, a sua ramificação e distribuição no parênquima ovariano.

\section{MATERIAIS E MÉTODOS}

Foram utilizados 34 pares de ovários de éguas (Equus caballus) sem raça definida, adultas, com idades diferentes e desconhecidas, procedentes de várias regiões do Estado de Minas Gerais e abatidas no Frigorífico Pomar (Araguari, MG).

Os animais foram abatidos, os ovários localizados e retirados juntamente com o útero e a porção cranial do canal vaginal, este material foi acondicionado em sacos plásticos e congelado. No laboratório foi feito o descongelamento das peças, em água corrente, por aproximadamente 24 horas e em seguida foram pesadas. Foram retirados os ovários com seus respectivos pedículos e mensurados com o auxílio de paquímetro digital (Digimess ${ }^{\circledR}$ ) no comprimento (eixo craniocaudal), na largura (eixo lateromedial) e na espessura (eixo dorsoventral).

A artéria ovárica foi dissecada, isolada e canulada antes que penetrasse no pedículo ovariano. Os vasos foram lavados injetando-se solução salina e posteriormente ar para expulsar a solução. Após a lavagem dos vasos foram injetados $2 \mathrm{~mL}$ de acetona para facilitar a progressão da massa de "vinyl" corada de vermelho para a confecção dos modelos arteriais.

O material foi mantido em água corrente por 24 horas e submetido ao processo de corrosão em solução de $\mathrm{H}_{2} \mathrm{SO}_{4}$ a $30 \%$, por 72 a 96 horas. Isolaram-se as amostras representativas da vascularização arterial dos órgãos, com jatos finos e controlados de água contra o parênquima, para retirá-lo.

Foi realizada foto-documentação de cada modelo, registrando a divisão da artéria ovárica e seus ramos, bem como a origem e localização dos vasos colaterais em relação aos quadrantes dorsocranial, ventrocranial, dorsocaudal e ventrocaudal. Esta localização foi obtida mediante o traçado de dois planos perpendiculares entre si, um entre as extremidades do órgão, delimitando as metades dorsal e ventral (x) e o outro no centro geométrico (y), demarcando as metades cranial e caudal.

Os resultados foram submetidos à análise estatística, aplicando-se o teste de Wilcoxon [44] para comparar as freqüências resultantes de amostras emparelhadas e o teste U de Mann-Whitney [44] para amostras independentes. Estes testes também foram aplicados para a comparação das medidas de comprimento, largura, espessura e peso. Na análise de correlação foi utilizado o Coeficiente de Correlação por Postos de Spearman. Os cálculos e a aplicação dos testes estatísticos foram efetuados por meio do programa SPSS para computadores.

\section{RESULTADOS}

Verificou-se que a artéria ovárica apresenta um trajeto longo, flexuoso e espiralado alcançando a glândula através da margem mesovárica, percorrendo-a em posição ora dorsal, ora ventral, em direção à extremidade tubárica, contornando-a até alcançar a fossa ovárica, penetrando no órgão após ter percorrido toda a sua superfície. Neste momento surgem dois arranjos vasculares, um a artéria permanece única até a entrada no parênquima (Figura $1 \mathrm{~A}$ e B) e outro se 
bifurca antes de penetrar no órgão (Figura $1 \mathrm{C}$ e D). Estes arranjos foram divididos em grupo A e B, respectivamente.

Grupo A: Em 51 preparações (75\%) a artéria ovárica emite de 2 a 62 ramos dorsais e de 4 a 46 ramos ventrais durante seu trajeto na margem mesovárica ao entrar no parênquima.
Em relação aos ovários direitos identificaramse como valores médio, mínimo e máximo, respectivamente: 39,0 (9,0 e 77,0) vasos colaterais; $1,5(0,0$ e 24,0$)$ distribuídos na face medial e 33,5 (9,0 e 62,0) na face lateral do órgão, onde os ramos dorsais resultaram em 17,0 (2,0 e 38,0) vasos colaterais e os ramos ventrais $20,5(4,0$ e 39,0$)$ vasos (Tabela 1$)$.

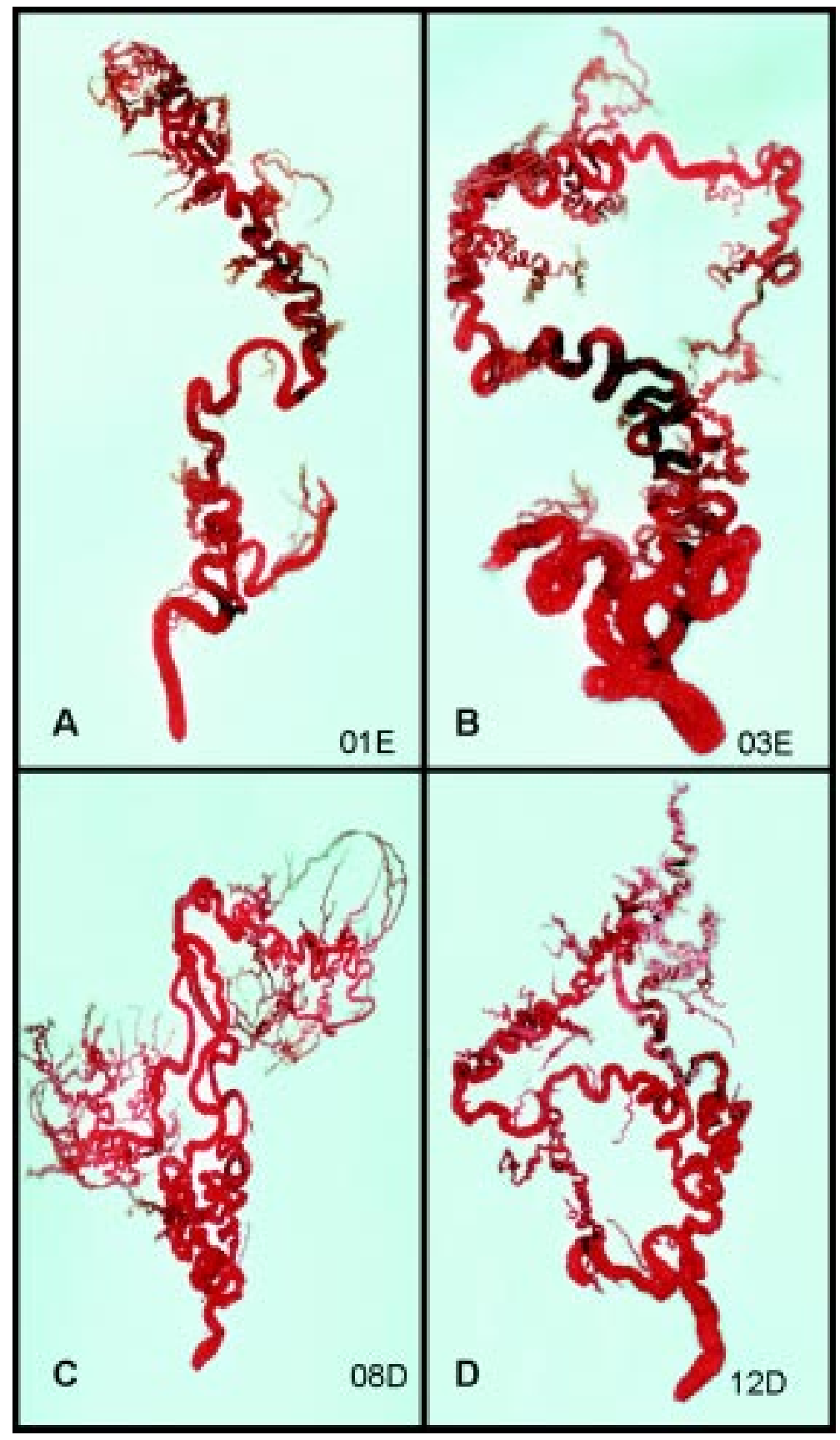

Figura 1. Fotografia do modelo "VINYL" representativa da vascularização arterial do ovário de égua S.R.D., mostrando: A e B - artéria ovárica com os respectivos colaterais, quando a artéria ovárica cede número variável de ramos dorsais e ventrais para a nutrição do órgão (Grupo A); C e D - divisão da artéria ovárica em um ramo dorsal e outro ventral ao nível da extremidade uterina (Grupo B). 
Cabral L.G., Facciotti P.R., Teixeira D.G., Araújo K.P.C., Martins D.S., Rici R.E.G., Ambrósio C.E., Miglino M.A. \& Mariana A.N.B. 2007. Comportamento da artéria ovárica em éguas sem raça definida...

Acta Scientiae Veterinariae. 35:345-355.

Quanto aos ovários esquerdos, identificaramse como valores médio, mínimo e máximo, respectivamente: $30,0(15,0$ e 92,0$)$ vasos colaterais, distribuídos em 2,0 (0,0 e 34,0) vasos na face medial e 27,5 $(12,0$ e 60,0$)$ na face lateral, onde os ramos dorsais fornecem 13,5 (3,0 e 62,0) vasos colaterais e os ramos ventrais $16,0(6,0$ e 46,0) (Tabela 2).

De acordo com a distribuição dos vasos colaterais dos ovários direitos nos quadrantes, registraram-se como valores médio, mínimo e máximo, res-

Tabela 1. Número total de ramos dorsais e ventrais da artéria ovárica, em éguas sem raça definida, distribuídas nas faces medial (M) e lateral (L), para a vascularização dos ovários direitos (D) (Grupo A) - Uberlândia (MG), 2002.

\begin{tabular}{|c|c|c|c|c|c|c|c|c|c|}
\hline \multirow{2}{*}{$\begin{array}{c}\begin{array}{c}\text { Ramos } \\
\text { arteriais }\end{array} \\
\text { Faces Obs. }\end{array}$} & \multicolumn{3}{|c|}{ Ramos dorsais da artéria ovárica } & \multicolumn{3}{|c|}{ Ramos ventrais da artéria ovárica } & \multicolumn{3}{|c|}{ Total } \\
\hline & $\mathbf{M}$ & L & то & $\mathbf{M}$ & L & TO & $M$ & L & TO \\
\hline 01D & 00 & 09 & 09 & 00 & 07 & 07 & 11 & 16 & 16 \\
\hline 02D & 01 & 16 & 17 & 05 & 18 & 23 & 06 & 34 & 40 \\
\hline 03D & 02 & 09 & 11 & 01 & 08 & 09 & 03 & 17 & 20 \\
\hline 04D & 00 & 10 & 10 & 03 & 11 & 14 & 03 & 21 & 24 \\
\hline 07D & 00 & 06 & 06 & 00 & 10 & 10 & 00 & 16 & 16 \\
\hline $11 \mathrm{D}$ & 00 & 15 & 15 & 00 & 20 & 20 & 00 & 35 & 35 \\
\hline $13 D$ & 00 & 14 & 14 & 00 & 07 & 07 & 00 & 21 & 21 \\
\hline $14 \mathrm{D}$ & 00 & 11 & 11 & 00 & 13 & 13 & 00 & 24 & 24 \\
\hline $15 \mathrm{D}$ & 13 & 25 & 38 & 02 & 37 & 39 & 15 & 62 & 77 \\
\hline 17D & 00 & 13 & 13 & 01 & 20 & 21 & 01 & 33 & 34 \\
\hline $18 \mathrm{D}$ & 00 & 02 & 02 & 00 & 07 & 07 & 00 & 09 & 09 \\
\hline $20 \mathrm{D}$ & 00 & 17 & 17 & 00 & 23 & 23 & 00 & 40 & 40 \\
\hline 21D & 00 & 11 & 11 & 00 & 22 & 22 & 00 & 33 & 33 \\
\hline 22D & 00 & 29 & 29 & 00 & 21 & 21 & 00 & 50 & 50 \\
\hline 23D & 15 & 07 & 22 & 05 & 21 & 26 & 20 & 28 & 48 \\
\hline $24 \mathrm{D}$ & 02 & 18 & 20 & 00 & 28 & 28 & 02 & 46 & 48 \\
\hline $25 \mathrm{D}$ & 02 & 22 & 24 & 00 & 19 & 19 & 02 & 41 & 43 \\
\hline $26 \mathrm{D}$ & 00 & 23 & 23 & 00 & 04 & 04 & 00 & 27 & 27 \\
\hline $28 \mathrm{D}$ & 00 & 31 & 31 & 11 & 18 & 29 & 11 & 49 & 60 \\
\hline $29 \mathrm{D}$ & 00 & 45 & 45 & 00 & 22 & 22 & 00 & 67 & 67 \\
\hline $30 \mathrm{D}$ & 01 & 23 & 24 & 04 & 12 & 16 & 05 & 35 & 40 \\
\hline 32D & 06 & 29 & 35 & 07 & 26 & 33 & 13 & 55 & 68 \\
\hline 33D & 09 & 05 & 14 & 12 & 16 & 28 & 21 & 21 & 42 \\
\hline $34 \mathrm{D}$ & 03 & 26 & 29 & 21 & 11 & 32 & 24 & 37 & 61 \\
\hline Total & 54 & 391 & 445 & 74 & 396 & 469 & 127 & 784 & 914 \\
\hline Média & 2,25 & 16,29 & 18,54 & 3,08 & 16,50 & 19,54 & 5,29 & 32,79 & 38,08 \\
\hline DP & 4,24 & 8,32 & 9,26 & 5,17 & 7,87 & 9,39 & 7,66 & 13,48 & 17,22 \\
\hline v & 17,98 & 69,22 & 85,75 & 26,73 & 61,94 & 88,17 & 58,68 & 181,71 & 296,53 \\
\hline ME & 0,0 & 15,5 & 17,0 & 0,5 & 17,5 & 20,5 & 1,5 & 33,5 & 39,0 \\
\hline Moda & 0 & 9 & 11 & 0 & 7 & 7 & 0 & 21 & 40 \\
\hline V. máx & 15 & 31 & 38 & 21 & 37 & 39 & 24 & 62 & 77 \\
\hline V. mín & 00 & 02 & 02 & 00 & 04 & 04 & 00 & 09 & 09 \\
\hline
\end{tabular}

To= total; $\mathrm{DP}=$ desvio-padrão; $\mathrm{V}=$ variância; $\mathrm{ME}=$ mediana; $\mathrm{V}$. máx= valor máximo e $\mathrm{V}$. min= mínimo obtido. 
Tabela 2. Número total de ramos dorsais e ventrais da artéria ovárica, em éguas sem raça definida, distribuídas nas faces medial (M) e lateral (L), para a vascularização dos ovários esquerdos (E) (Grupo A) - Uberlândia (MG), 2002.

\begin{tabular}{|c|c|c|c|c|c|c|c|c|c|}
\hline \multirow{2}{*}{$\begin{array}{c}\begin{array}{c}\text { Ramos } \\
\text { arteriais }\end{array} \\
\text { Faces Obs. }\end{array}$} & \multicolumn{3}{|c|}{ Ramos dorsais da artéria ovárica } & \multicolumn{3}{|c|}{ Ramos ventrais da artéria ovárica } & \multicolumn{3}{|c|}{ Total } \\
\hline & M & $\mathbf{L}$ & TO & M & $\mathbf{L}$ & TO & $\mathbf{M}$ & $\mathbf{L}$ & Tо \\
\hline $01 \mathrm{E}$ & 02 & 15 & 17 & 00 & 13 & 13 & 02 & 28 & 30 \\
\hline 02E & 02 & 19 & 21 & 05 & 08 & 13 & 07 & 27 & 34 \\
\hline 03E & 00 & 14 & 14 & 04 & 12 & 16 & 04 & 26 & 30 \\
\hline 04E & 04 & 10 & 14 & 00 & 10 & 10 & 04 & 20 & 24 \\
\hline 07E & 00 & 12 & 12 & 00 & 09 & 09 & 00 & 21 & 21 \\
\hline 09E & 03 & 24 & 27 & 01 & 22 & 23 & 04 & 46 & 50 \\
\hline $10 \mathrm{E}$ & 00 & 13 & 13 & 00 & 13 & 13 & 00 & 26 & 26 \\
\hline $11 \mathrm{E}$ & 00 & 03 & 03 & 04 & 12 & 16 & 04 & 15 & 19 \\
\hline $12 \mathrm{E}$ & 00 & 10 & 10 & 08 & 23 & 31 & 08 & 33 & 41 \\
\hline $13 \mathrm{E}$ & 00 & 06 & 06 & 01 & 15 & 16 & 01 & 21 & 22 \\
\hline $14 \mathrm{E}$ & 00 & 04 & 04 & 02 & 09 & 11 & 02 & 13 & 15 \\
\hline $15 \mathrm{E}$ & 15 & 17 & 32 & 19 & 26 & 45 & 34 & 43 & 77 \\
\hline $16 \mathrm{E}$ & 00 & 10 & 10 & 02 & 12 & 14 & 02 & 22 & 24 \\
\hline $17 \mathrm{E}$ & 00 & 12 & 12 & 00 & 06 & 06 & 00 & 18 & 18 \\
\hline $18 \mathrm{E}$ & 03 & 05 & 08 & 00 & 07 & 07 & 03 & 12 & 15 \\
\hline $20 \mathrm{E}$ & 00 & 26 & 26 & 00 & 25 & 25 & 00 & 51 & 51 \\
\hline $22 \mathrm{E}$ & 00 & 25 & 25 & 02 & 15 & 17 & 02 & 40 & 42 \\
\hline $24 \mathrm{E}$ & 00 & 11 & 11 & 00 & 09 & 09 & 00 & 20 & 20 \\
\hline $25 \mathrm{E}$ & 00 & 13 & 13 & 02 & 15 & 17 & 02 & 28 & 30 \\
\hline $27 \mathrm{E}$ & 00 & 05 & 05 & 00 & 10 & 10 & 00 & 15 & 15 \\
\hline $28 \mathrm{E}$ & 00 & 20 & 20 & 01 & 17 & 18 & 01 & 37 & 38 \\
\hline $29 \mathrm{E}$ & 07 & 45 & 52 & 02 & 14 & 16 & 09 & 59 & 68 \\
\hline $30 \mathrm{E}$ & 01 & 24 & 25 & 00 & 34 & 34 & 01 & 58 & 59 \\
\hline $31 \mathrm{E}$ & 00 & 11 & 11 & 01 & 32 & 33 & 01 & 43 & 44 \\
\hline $32 \mathrm{E}$ & 00 & 17 & 17 & 00 & 22 & 22 & 00 & 39 & 39 \\
\hline $33 \mathrm{E}$ & 31 & 31 & 62 & 01 & 29 & 30 & 32 & 60 & 92 \\
\hline $34 \mathrm{E}$ & 00 & 23 & 23 & 01 & 16 & 17 & 01 & 39 & 40 \\
\hline Total & 68 & 426 & 494 & 56 & 435 & 492 & 124 & 861 & 985 \\
\hline Média & 2,62 & 15,78 & 18,3 & 2,07 & 16,11 & 18,22 & 4,59 & 31,89 & 36,48 \\
\hline DP & 6,52 & 9,48 & 13,59 & 3,88 & 7,82 & 9,57 & 8,55 & 14,56 & 19,69 \\
\hline v & 42,57 & 89,95 & 184,6 & 15,07 & 61,1 & 91,56 & 73,17 & 211,87 & 387,8 \\
\hline ME & 0,0 & 13,0 & 14,0 & 1,0 & 14,0 & 16,0 & 2,0 & 28,0 & 30,0 \\
\hline Moda & 0 & 10 & 10 & 0 & 9 & 16 & 0 & 15 & 15 \\
\hline V. máx & 31 & 45 & 62 & 19 & 34 & 46 & 34 & 60 & 92 \\
\hline V. mín & 00 & 03 & 03 & 00 & 06 & 06 & 00 & 12 & 15 \\
\hline
\end{tabular}

$\mathrm{To}=$ total; DP= desvio-padrão; V= variância; ME= mediana; V. máx= valor máximo e V. min= mínimo obtido. 
pectivamente: dorsocranial $10,5(0,0$ e 29,0$)$ vasos colaterais; dorsocaudal 7,0 (0,0 e 28,0) vasos; ventrocranial 9,5 (2,0 e 33,0) vasos; e ventrocaudal com $6,5(0,0$ e 24,0$)$ vasos. Dos quadrantes dorsais 0,0 $(0,0$ e 20,0$)$ vasos se apresentam na face medial e 16,5 (3,0 e 37,0) na face lateral; dos quadrantes ventrais $0,0(0,0$ e 21,0$)$ na face medial e $16,0(4,0$ e 37,0) na face lateral do ovário.

Em relação à distribuição dos vasos colaterais dos ovários esquerdos identificaram-se como valores médio, mínimo e máximo, respectivamente: dorsocranial 7,0 (0,0 e 35,0) vasos colaterais; dorsocaudal $4,0(0,0$ e 29,0$)$ vasos colaterais; ventrocranial 10,0 $(4,0$ e 28,0$)$; e ventrocaudal com $8,0(0,0$ e 18,0$)$ vasos colaterais. Dos quadrantes dorsais $0,0(0,0 \mathrm{e}$ $31,0)$ vasos se apresentam na face medial e $12,0(2,0$ e 35,0$)$ vasos na face lateral; dos quadrantes ventrais $1,0(0,0$ e 10,0$)$ vasos se apresentam na face medial e 15,5 (6,0 e 35,0) na face lateral da glândula.

Neste grupo, em 43 preparações, observou-se que os vasos colaterais, oriundos do ramo dorsal, além de fornecerem vasos aos quadrantes correspondentes, contribuem com 1 a 27 vasos para os quadrantes ventrais; enquanto os ramos ventrais, em 41 das preparações, contribuem com 1 a 21 vasos para os quadrantes dorsais.

Grupo B: Este grupo refere-se a 17 preparações $(25 \%)$. A artéria ovárica na extremidade uterina divide-se em ramos dorsal e ventral. O ramo dorsal emite de 6 a 31 vasos colaterais para os ovários direitos (10 preparações) e 6 a 28 vasos colaterais para os ovários esquerdos (7 preparações). O ramo ventral cede de 2 a 33 vasos colaterais para os ovários direitos (10 preparações) e 14 a 34 vasos colaterais para os ovários esquerdos (7 preparações) (Tabelas 3 e 4).

Quanto ao número total de vasos colaterais para os ovários direitos neste grupo determinaramse como valores médio, mínimo e máximo, respectivamente: $26,0(17,0$ e 62,0$)$ vasos, onde $1,0(0,0$ e $21,0)$ vaso encontra-se na face medial e $25,0(17,0 \mathrm{e}$ $41,0)$ na face lateral. O ramo dorsal participa com $14,5(6,0$ e 31,0) vasos colaterais e o ramo ventral dá

Tabela 3. Número total de ramos dorsais e ventrais da artéria ovárica, em éguas sem raça definida, distribuídas nas faces medial (M) e lateral (L), para a vascularização dos ovários direitos (D) (Grupo B) - Uberlândia (MG), 2002.

\begin{tabular}{|c|c|c|c|c|c|c|c|c|c|c|}
\hline \multirow{2}{*}{$\begin{array}{c}\begin{array}{c}\text { Ramos } \\
\text { arteriais }\end{array} \\
\text { Faces Obs. }\end{array}$} & \multicolumn{3}{|c|}{ Ramos dorsais da artéria ovárica } & \multicolumn{3}{|c|}{ Ramos ventrais da artéria ovárica } & \multicolumn{3}{|c|}{ Total } & \multirow{2}{*}{ Adicionais } \\
\hline & M & $\mathbf{L}$ & TO & M & $\mathbf{L}$ & TO & M & $\mathbf{L}$ & TO & \\
\hline 05D & 08 & 23 & 31 & 00 & 02 & 02 & 08 & 25 & 33 & 00 \\
\hline 06D & 00 & 07 & 07 & 00 & 10 & 10 & 00 & 17 & 17 & 00 \\
\hline 08D & 14 & 15 & 29 & 07 & 26 & 33 & 21 & 41 & 62 & 00 \\
\hline 09D & 00 & 14 & 14 & 00 & 13 & 13 & 00 & 27 & 27 & 00 \\
\hline $10 \mathrm{D}$ & 00 & 15 & 15 & 02 & 08 & 10 & 02 & 23 & 25 & 00 \\
\hline $12 \mathrm{D}$ & 00 & 18 & 18 & 00 & 07 & 07 & 00 & 25 & 25 & 00 \\
\hline $16 \mathrm{D}$ & 02 & 09 & 11 & 00 & 22 & 22 & 02 & 31 & 33 & 00 \\
\hline $19 D$ & 00 & 06 & 06 & 00 & 13 & 13 & 00 & 19 & 19 & 00 \\
\hline 27D & 02 & 16 & 18 & 00 & 11 & 11 & 02 & 27 & 29 & 00 \\
\hline 31D & 00 & 15 & 15 & 00 & 10 & 10 & 00 & 25 & 25 & 00 \\
\hline Total & 26 & 138 & 144 & 09 & 122 & 131 & 35 & 260 & 295 & 02 \\
\hline Média & 2,60 & 13,80 & 14,4 & 0,9 & 12,20 & 13,10 & 3,50 & 26,00 & 29,50 & 0,20 \\
\hline DP & 4,72 & 5,18 & 7,21 & 2,23 & 7,05 & 8,62 & 6,62 & 6,62 & 12,54 & 0,63 \\
\hline V & 22,28 & 26,83 & 51,98 & 4,97 & 49,70 & 74,30 & 43,78 & 43,78 & 157,25 & 0,40 \\
\hline ME & 0,0 & 15,0 & 14,5 & 0,0 & 10,5 & 10,5 & 1,0 & 25,0 & 26,0 & 0,0 \\
\hline Moda & 0 & 15 & 15 & 0 & 10 & 10 & 0 & 25 & 25 & 0 \\
\hline V. máx & 14 & 23 & 31 & 7 & 26 & 33 & 21 & 41 & 62 & 02 \\
\hline V. mín & 00 & 06 & 06 & 00 & 02 & 02 & 00 & 17 & 17 & 00 \\
\hline
\end{tabular}


Tabela 4. Número total de ramos dorsais e ventrais da artéria ovárica, em éguas sem raça definida, distribuídas nas faces medial (M) e lateral (L), para a vascularização dos ovários esquerdos (E) (Grupo B) - Uberlândia (MG), 2002.

\begin{tabular}{|c|c|c|c|c|c|c|c|c|c|c|}
\hline \multirow{2}{*}{$\begin{array}{c}\begin{array}{c}\text { Ramos } \\
\text { arteriais }\end{array} \\
\text { Faces Obs. }\end{array}$} & \multicolumn{3}{|c|}{ Ramos dorsais da artéria ovárica } & \multicolumn{3}{|c|}{ Ramos ventrais da artéria ovárica } & \multicolumn{3}{|c|}{ Total } & \multirow{2}{*}{ Adicionais } \\
\hline & M & $\mathbf{L}$ & TO & M & $\mathbf{L}$ & TO & M & $\mathbf{L}$ & TO & \\
\hline $05 \mathrm{E}$ & 00 & 12 & 12 & 03 & 14 & 17 & 03 & 26 & 29 & 00 \\
\hline $06 \mathrm{E}$ & 00 & 06 & 06 & 00 & 18 & 18 & 00 & 24 & 24 & 00 \\
\hline $08 \mathrm{E}$ & 03 & 03 & 06 & 00 & 14 & 14 & 03 & 17 & 20 & 00 \\
\hline $19 \mathrm{E}$ & 05 & 16 & 21 & 00 & 22 & 22 & 05 & 38 & 43 & 00 \\
\hline $21 \mathrm{E}$ & 00 & 12 & 12 & 00 & 30 & 30 & 00 & 42 & 42 & 00 \\
\hline $23 E$ & 13 & 05 & 28 & 05 & 29 & 34 & 18 & 44 & 62 & 00 \\
\hline $26 \mathrm{E}$ & 03 & 06 & 09 & 09 & 17 & 26 & 12 & 23 & 35 & 00 \\
\hline Total & 24 & 70 & 94 & 17 & 144 & 161 & 41 & 214 & 255 & 00 \\
\hline Média & 3,43 & 10,00 & 13,43 & 2,43 & 20,57 & 23,00 & 5,86 & 30,57 & 36,43 & 0,00 \\
\hline DP & 4,65 & 5,00 & 8,20 & 3,51 & 6,68 & 7,33 & 6,72 & 10,58 & 14,20 & 0,00 \\
\hline V & 21,62 & 25,00 & 67,28 & 12,29 & 44,62 & 53,67 & 45,14 & 111,95 & 201,62 & 0,00 \\
\hline ME & 3,0 & 12,0 & 12,0 & 0,0 & 18,0 & 22,0 & 3,0 & 26,0 & 35,0 & 0,0 \\
\hline Moda & 0 & 6 & 6 & 0 & 14 & 14 & 0 & 17 & 20 & 0 \\
\hline V. máx & 13 & 16 & 28 & 09 & 30 & 34 & 18 & 44 & 62 & 00 \\
\hline V. mín & 00 & 03 & 06 & 00 & 14 & 14 & 00 & 17 & 20 & 00 \\
\hline
\end{tabular}

To= total; $\mathrm{DP}=$ desvio-padrão; $\mathrm{V}=$ variância; $\mathrm{ME}=$ mediana; $\mathrm{V}$. máx= valor máximo e V. min= mínimo obtido.

origem a $10,5(2,0$ e 33,0$)$ vasos colaterais. Em uma das preparações (obs. 31D) a artéria ovárica emite, antes da sua divisão em ramos dorsal e ventral, 2 ramos adicionais (Tabela 3).

Em relação aos ovários esquerdos, encontraram-se como valores médio, mínimo e máximo, respectivamente: $35,0(20,0$ e 62,0$)$ vasos colaterais, sendo 3,0 (0,0 e 18,0) na face medial e 26,0 (17,0 e $44,0)$ na face lateral do órgão. O ramo dorsal participa com $12,0(6,0$ e 28,0$)$ e o ramo ventral com 22,0 $(14,0$ e 34,0$)$ vasos colaterais (Tabela 4).

Com relação à distribuição dos vasos colaterais pelos quadrantes dos ovários direitos registraram-se, como valores médio, mínimo e máximo, respectivamente: os quadrantes dorsais $14,0(7,0$ e 30,0$)$ vasos colaterais [dorsocranial com 7,0 $(0,0$ e 14,0) e o dorsocaudal 7,5 (3,0 e 19,0)], distribuídos em 13,0 $(7,0$ e 23,0$)$ pela face lateral e $0,0(0,0$ e 14,0$)$ pela face medial; os quadrantes ventrais $13,0(4,0$ e 32,0$)$ vasos colaterais [ventrocranial com 7,5 (1,0 e 29,0) e ventrocaudal com 4,0 (1,0 e 10,0)], distribuídos $12,0(4,0$ e 25,0$)$ na face lateral e $0,0(0,0$ e 7,0$)$ na face medial do órgão.

$\mathrm{Na}$ distribuição dos vasos colaterais pelos quadrantes dos ovários esquerdos observou-se nos quadrantes dorsais $25,0(10,0$ e 39,0$)$ vasos colaterais [dorsocranial com 12,0 (4,0 e 30,0) e dorsocaudal com 10,0 (3,0 e 15,0)], distribuídos em 17,0 (7,0 e $39,0)$ vasos pela face lateral e 3,0 $(0,0$ e 12,0) pela face medial. Os quadrantes ventrais apresentaram 9,0 $(3,0$ e 37,0$)$ vasos colaterais [ventrocranial com 6,0 $(2,0$ e 20,0$)$ e ventrocaudal com $3,0(0,0$ e 17,0$)]$, sendo $9,0(3,0$ e 27,0$)$ vasos na face lateral e $0,0(0,0$ e 10,0$)$ na face medial da glândula.

Verificou-se neste grupo que a artéria ovárica cede ramos dorsais em 8 preparações, contribuindo com 1 a 17 vasos aos quadrantes ventrais; enquanto que os ramos ventrais e seus colaterais, em 14 preparações, contribuem com 1 a 27 vasos aos quadrantes dorsais.

No estudo dos modelos arteriais da vascularização dos ovários registrou-se, sem levar em consideração os diferentes grupos, para os ovários direitos um total de 33,0 $(9,0$ e 77,0) vasos colaterais, dos quais $27,5(9,0$ e 63,0$)$ encontram-se na face lateral e 1,5 $(0,0$ e 24,0$)$ na face medial; a distribuição destes vasos nos quadrantes demonstra: no dorsocranial 8,5 $(0,0$ e 29,0$)$ vasos, no dorsocaudal $7,0(1,0$ e 28,0$)$ vasos, no ventrocranial $8,0(1,0$ e 33,0$)$ vasos, ventrocaudal $5,5(0,0$ e 24,0$)$ vasos, craniais $18,5(6,0$ e 46,0$)$ vasos, 
caudais 12,5 (2,0 e 38,0) vasos, dorsais $16,0(3,0$ e $46,0)$ vasos e ventrais com $16,5(4,0$ e 40,0$)$ vasos.

Os ovários esquerdos apresentam no total $32,0(15,0$ e 92,0$)$ vasos colaterais, sendo $26,0(12,0$ e 60,0) na face lateral e $2,0(0,0$ e 32,0) na face medial. Desta forma, os vasos nos diversos quadrantes estão distribuídos da forma seguinte: 8,5 (1,0 e 35,0) dorsocranial, 6,0 (0,0 e 29,0) dorsocaudal, 9,0 (2,0 e 28,0) ventrocranial e $6,0(0,0$ e 18,0$)$ no quadrante ventrocaudal.

Pelas medidas realizadas nos eixos craniocaudal (comprimento), lateromedial (largura) e dorsoventral (espessura) dos ovários direito e esquerdo, obteve-se uma média de 43,71mm, 45,29mm, 34,11 $\mathrm{mm}, 38,74 \mathrm{~mm}, 24,78 \mathrm{~mm}, 27,79 \mathrm{~mm}$, respectivamente. O peso dos ovários resultou, em média, de 45,67g para os ovários direitos e 52,21g para os ovários esquerdos.

\section{Análise estatística}

Com o objetivo de verificar a existência ou não de diferenças significantes entre as frequiências de ramos encontrados nos ovários direito e esquerdo, foi aplicado o teste não paramétrico [44]. O nível de significância foi estabelecido em 0,05 em uma prova bilateral. Foram encontradas diferenças significativas nas variáveis lateral-ventral e total-ventral, e as freqüências mais elevadas, nos dois casos, foram às relativas aos ovários esquerdos para o grupo $\mathrm{B}$.

Entre os grupos A e B identificamos diferenças significantes nos resultados, os quais mostraram em relação aos vasos colaterais diferenças significativas somente aos a frequiência de vasos dorsais do ovário esquerdo, e as frequiências mais elevadas foram às relativas aos animais do grupo $\mathrm{A}$.

Em relação às diferenças de freqüência entre os quatro quadrantes observaram-se em ambos ovários, diferenças significativas relativas aos quadrantes dorsocranial e ventrocranial. No grupo A as freqüências mais elevadas com relação à face lateral foram relativas ao quadrante caudal, e na face medial foram às relativas ao quadrante cranial. No grupo B diferenças significativas foram encontradas somente entre as frequiências totais sendo as mais elevadas relativas à porção cranial. Através de analise de correlação foram consideradas como positivas e significantes entre todas as variáveis analisadas. Diferenças significativas foram encontradas entre as medidas dos ovários direito e esquerdo com relação à largura e à espessura, sendo que os valores mais elevados foram os obtidos com os ovários esquerdos.

\section{DISCUSSÃO}

Na literatura consultada, com relação à denominação atribuída à artéria responsável pela nutrição dos ovários, encontram-se diversas nomenclaturas. Algumas denominações encontradas, entre outras, foram: "artéria espermática interna" [17,43], "artéria útero-ovárica" [2,7,8,20,25, 34,37,39] e "artéria ovárica" [9]. Neste trabalho utiliza-se a nomenclatura "artéria ovárica" [28], denominação também usada por outros autores $[5,15,16,26,31,35,36]$.

Em relação à descrição do trajeto da artéria ovárica na superfície do órgão, foram constatadas divergências entre os autores consultados, o que deve estar relacionado com as diversas denominações atribuídas aos diferentes segmentos do ovário, por exemplo, pelo pólo superior [10] e borda superior [4]. Adotou-se a nomenclatura indicada pelo Internacional Committee On Veterinary Gross Anatomical Nomenclature [28], isto é, extremidades tubárica e uterina; margens mesovárica e livre, também utilizadas por outros autores [1,38,45].

Verificou-se que a artéria ovárica exibe um trajeto longo, sinuoso, espiralado e irregular, o que está em concordância com outros autores [1,27,31,38,43].

Os tratadistas quando descrevem a divisão da artéria ovárica em seus ramos principais citam apenas que estas artérias emitem vasos, sem caracterizá-los $[6,38,45,46]$. Alguns mencionam que a artéria ovárica emite dois ramos: tubárico e uterino [38], ovárico e uterino [34,35,37], ovárico e tubárico [29], artéria ovárica e artéria uterina cranial [8,27], não permitindo qualquer tipo de comparação com os resultados obtidos neste estudo.

Encontraram-se várias designações quanto às ramificações principais da artéria ovárica, que variam na dependência da divisão, calibre e posição por elas ocupados. Algumas destas denominações são: ramo ovárico e ramo uterino [16,17,25,34,35,37]; ramo ovariano e ramo uterino [12,23,24,32,33,40]; ramos cranial e caudal [30,31]; ramo tubárico e ramo uterino [28,38], entre outros. Nas preparações deste trabalho estes vasos foram identificados e designados como "ramos dorsais", "ramos ventrais" e "ramos adicionais". Aos vasos que se originam nestes ramos e se destinam ao parênquima da glândula atribuiu-se a denominação 
"vasos colaterais", à semelhança de outros autores $[1,19,20]$.

Observou-se nesta pesquisa que a artéria ovárica emite vários ramos para a superfície antes de entrar no parênquima da glândula, corroborando com relato da localização externa da porção vascular em ovários de éguas, em aposição à situação externa em outras espécies domésticas [22].

Com relação ao comprimento dos ovários, notou-se que os ovários esquerdos apresentam uma distribuição dos dados amostrais, levemente deslocados para a esquerda, enquanto os direitos tiveram valores mais próximos de uma distribuição normal.

Quanto à largura dos ovários, verificou-se que as medidas obtidas do ovário direito estão distribuídas de forma mais próxima dos valores normais do que as do ovário esquerdo, no qual a distribuição dos dados amostrais está levemente deslocada para a esquerda.

Quanto ao peso, os ovários esquerdos apresentaram maior desvio em relação à média, deslocando-se levemente para a esquerda.

Quanto à espessura dos ovários, os dados apresentam uma pequena dispersão em relação à distribuição normal dos ovários esquerdos. Os valores de espessura, dentre todos os parâmetros analisados, foram os que apresentaram uma dispersão menor, e estes são menos heterogêneos, aspectos não abordados por nenhum dos autores consultados.

O trajeto sinuoso, espiralado e irregular da artéria ovárica, fora e dentro do parênquima ovariano, pode estar relacionado às diversas funções, como uma adaptação da vascularização e crescimento ovariano, atividade estrogênica do órgão e existência de um mecanismo de equalização da pressão sanguínea no interior da glândula [41,42].

Outro aspecto importante é a relação entre a artéria e veia ovárica, em que autores [11,12,14,16,18,2124,33,40] são unânimes ao afirmar que na espécie eqüina tal contato é mínimo, podendo estar relacionado à presença de um efeito lúteo-lítico útero-ovariano local nas ovelhas, porcas e vacas e a aparente ausência deste efeito local em éguas.

\section{CONCLUSÕES}

A artéria ovárica exibe um trajeto longo, flexuoso e espiralado, alcança a glândula por meio da margem mesovárica, corre em posição ora dorsal ora ventral, percorre a margem mesovárica em direção à extremidade tubárica, contorna-a até alcançar a fossa ovárica, após ter percorrido toda a sua superfície e penetra no órgão.

Existem dois tipos de arranjos vasculares, ou seja, a artéria ovárica emite ramos alternados $(75 \%$ das amostras estudadas) ou bifurca-se na extremidade uterina em ramos dorsal e ventral (25\% das amostras estudadas) e antes possui dois ramos adicionais.

Os ovários direitos apresentam um número maior de ramos, 33,0 (9,0 e 77,0 vasos), que os esquerdos, 32,0 (15,0 e 92,0 vasos), porém são menores, em todos os parâmetros analisados. A face lateral dos ovários concentra maior número de ramos em relação à face medial.

Nos ovários direitos, os quadrantes mais irrigados, por ordem, são: o dorsocranial, o ventrocranial, o dorsocaudal e o ventrocaudal. Nos ovários esquerdos, observa-se uma inversão, isto é, os quadrantes mais irrigados são por ordem: o ventrocranial, o dorsocranial, o ventrocaudal e o dorsocaudal.

\section{REFERÊNCIAS}

1 Barone R. 1990. Anatomie Comparée des Mammifères Domestiques. Paris: Édition Vigot, 389p.

2 Barone R. \& Burel H. 1957. Les Vaisseaux Sangüins du Tractus Genital Chez la Vache. Revue de Medicine Vétérinaire Toulouse. 108: 382-395.

3 Barone R., Frapart P. \& Pavaux C.L. 1962. Les Vaisseaux Sanguins de L'appareil Génital Chez la Truie. Bulletin de la Société des Sciences Vétérinaires de Lyon. 64: 337-346.

4 Barone R. \& Pavaux C.L. 1962. Les Vaisseaux Sangüins du Tractus Genital Chez les Femelles Domestiques. Bulletin de la Société des Sciences Vétérinaires de Lyon.1: 3-51.

5 Belou P. 1934. Revision Anatomica del Sistema Arterial, III. Atlas Esterescopica de Anatomia de las Artérias; segunda parte; pp.114 (Estereos 215). Buenos Aires, apud Delson B., Lubin S. \& Reynolds S.R.M. 1949. Vascular Patternals in the Human Ovary. American Journal of Obstetric and Gynecology. 57: 842-853.

6 Bourdelle E. 1920. Anatomie Régionale des Animaux Domestiques. v.3. Paris : J.B. Baillière, pp.321-323.

7 Bressou M.C. \& Gall J.L. 1936. Contribution à l'étude de la vascularisation de l'uterus dês ruminants. Recueil de Medicine Vétérinaire. 112: 5-9. 
Cabral L.G., Facciotti P.R., Teixeira D.G., Araújo K.P.C., Martins D.S., Rici R.E.G., Ambrósio C.E., Miglino M.A.\& Mariana A.N.B. 2007.

8 Bruni A.C. \& Zimmerl U. 1947. Anatomia degli animali domestici. 2nd edn. Milano: Francesco Vallardi, pp.364-365.

9 Bruni A.C. \& Zimmerl U. 1977. Anatomia degli animali domestici. 2nd edn. Milano: Francesco Vallardi, 352p.

10 Dawson A.B. \& Reis J.H. 1922. An Anomalous Arterial Supply to Suprarenal, Kidney and Ovary. The Anatomical Record. 3: $161-167$.

11 Del Campo C.H. \& Ginther O.J. 1972. Anatomy of utero-ovarian vasculature of Mares, Ewes and Sows. Journal of Animal Science. 35 (Suppl 5): 1119p.

12 Del Campo C.H. \& Ginther O.J. 1973. Vascular Anatomy of the uterus and ovaries and the unilateral luteolytic effect of the uterus: Horses, Sheep and Swine. American Journal of Veterinary Research. 34 (Suppl 3): 305-316.

13 Dellmann H.D. \& Brown E.M. 1976. Textbook of Veterinary Histology. Philadelphia : Lea \& Febiger, 344p.

14 Dobrowolski W. \& Hafez E.S.E. 1970. Ovariouterine Vasculature in Sheep. American Journal of Veterinary Research. 31 : 21-26.

15 Drouet M.E. 1983. Um quarto tipo de Arcada Anastomótica entre as artérias ovárica e uterina na Mulher Negra Brasileira (Arco Anastomótico Arterial do Mesométrio). Revista de Ciências Biomédicas. 4: 55-59.

16 Dyce K.M., Sack W.O. \& Wensing C.J.C. 1990. Tratado de Anatomia Veterinária. Rio de Janeiro: Guababara Koogan S/A, $358 \mathrm{p}$.

17 Ellenberger W. \& Baum H. 1943. Handbuch der vergleichenden anatomie der haustiere. Berlin : Springer Verlag, 487p.

18 Enriquez-Yap E.L. 1975. Some observations on the extramural and intramural vascular supply patterns of the uteri and varies of Philippine carabaos (Bubalis bubalis). The Philippine Journal of Veterinary Medicine. 14: 1-16.

19 Esperança Pina J.A. \& Reis A.M. 1984. Arterial Component of the Angioarchitecture of the canine ovary. Acta Anatômica. 120: 112-116.

20 Favilli N. 1931. Nozione Comparate di Anatomia e Fisiologia degli Animal Rurali. Torino: Torinese, pp.400-401.

21 Ghazi R. 1981. Angioarchitectural studies of the Camel (Camellus dromedarius). Journal of Reproduction and Fertility. 61: 43-46.

22 Ginther O.J. 1976. Comparative Anatomy of utero-ovarian Vasculature. Veterinary Scope. 2: 1-17.

23 Ginther O.J. \& Del Campo C.H. 1974. Vascular Anatomy of the Uterus and Ovaries and the Unilateral Luteolytic Effect of the Uterus: Cattle. American Journal of Veterinary Research. 35: 193-203.

24 Ginther O.J., Garcia M.C., Squires E.L. \& Steffenhagen W.P. 1972. Anatomy of vasculature of uterus and ovaries in the Mare. American Journal of Veterinary Research. 33: 1561-1568.

25 Gonzalez Y Garcia J. \& Gonzales Alvarez R.G. 1961. Anatomia comparada de los animales domésticos. 7th edn. Madrid: Canales, pp.634-636.

26 Habel R.E. 1967. Anatomia de Disseccion de los Ruminantes Domésticos. Zaragoza : Acribia, pp. 69: 71-85.

27 Hafez E.S.E. 1993. Reproduction in farm animals. 6th edn. Philadelphia: Lea \& Febiger, 573p.

28 International Committee on Veterinary Gross Anatomical Nomenclature. 1994. Nômina Anatômica Veterinária. 4th edn. Zurich : Library of Congress, pp.125-133.

29 Koch T. 1963. Lehrbuch der Veterinar Anatomie. Jena: Gustav Fischer, pp.299-300.

30 König H.E. \& Ries R. 1987. Corrosion Anatomical - Studies of the blood Vessel system of the Mare Ovary. Tierarztl Prax. 15: 181-184.

31 König H.E. 1995. ZurAnatomie Des Eierstockesw der Stute. Acta Veterinária Brno. 64: 13-16.

32 Lamond D.R. \& Drost M. 1974. Blood supply to the bovine ovary. Journal of Animal Science. 38: 106-112.

33 Lee C.S. \& O'Shea J.D. 1976. The Extrinsic Blood Vessels of the Ovary of the Sheep. Journal of Morphology. 148: $287-304$.

34 Lesbre F.X. 1923. Précis D’Anatomia Comparée des Animaux Domestiques. v.4. Paris: J.B. Baillière, pp.374-375.

35 Mannu A. 1930. Aparecchio vascolare. In: Zimmerl U. Tratado de Anatomia Veterinária. Milano: Francesco Vallardi, pp.216-223.

36 May N.D.S. 1964. The Anatomy of the Sheep. 2nd edn. Brisbane: University of Queensland Press, pp.96-127.

37 Mcleod W.M. 1958. Bovine Anatomy. 2nd edn. Minneapolis: Burgess, pp.148-150.

38 Nickel R., Schummer A.S. \& Seiferle E. 1979. The viscera of the domestic mammals. Berlin: Paul Parey, pp.385-386.

39 Nunes Q. 1964. Blood Vessels of the Genitália and Acessory Genital Organs of Swine (Sus scrofa domesticus). 122p. Ames, Iowa. Thesis, Iowa State University of Science And Technology.

40 Panchamukhi B.G. \& Mudholkar D.R. 1973. Study of the Arteries and veins (angioarchitecture) of the Internal Genitalia of sheep and goats with particular reference to the ovaries. The Indian Journal Animal Science. 43: 1057-1060. 
41 Reynolds S.R.M. 1947. Adaptation of the Spiral artery in the rabbit ovary to changes in organize after stimulations by gonadotrophins; effect of ovulation and luteinization. Endocrinology. 40: 381-387.

42 Reynolds S.R.M. 1947. Distortion of the Spiral artery in the ovary associated with corpus hemorrhagicun cysts. Endocrinology. 40: 388-394.

43 Schwarze E. \& Schröder L. 1981. Compêndio de Anatomia Veterinária. Zaragoza : Acribia, 72p.

44 Siegel S. 1975. Estatística Não-paramétrica para as Ciências do Comportamento. Ed. McGraw-Hill do Brasil, São Paulo, $350 \mathrm{p}$.

45 Sisson S. 1981. Aparelho urogenital do Eqüino. In:.Getty R. Sisson/Grossman (Ed). Anatomia dos Animais Domésticos. 5th ed. Rio de Janeiro: Interamericana, pp.507-512.

46 Zuckerman S. \& Baker T.G. 1977. The development of the ovary and the process of oogenesis. In: Zuckerman S. \& Weir B.J. (Eds). The Ovary. New York: Academic Press, pp.41-67. 